\title{
Das Instrument der Mehrjahresprogramme in der europäischen Justiz- und Innenpolitik
}

\author{
Jörg Semmler*
}

Der Zyklus des sogenannten Haager Programms ${ }^{1}$ (2005-2009), des justiz- und innenpolitischen Handlungsprogramms der Europäischen Union, neigt sich seinem Ende entgegen. Der Bereich der europäischen Justiz- und Innenpolitik ist einer der jüngsten und zugleich politisch bedeutsamsten Integrationsbereiche der Europäischen Union. Themen wie die Bekämpfung illegaler Migration, organisierter Kriminalität und des Terrorismus, die grenzüberschreitende polizeiliche und justizielle Zusammenarbeit, die Harmonisierung von Strafund Strafverfahrensrecht, der Verfügbarkeitsgrundsatz beim polizeilichen Informationsaustausch oder die Integration von Migranten berühren unmittelbar die Souveränität der Mitgliedstaaten. Die Europäische Union hat in diesem Bereich mit den beiden bisherigen Mehrjahresprogrammen von Tampere ${ }^{2}$ und Den Haag positive Erfahrungen gemacht. Der Implementierungsstand des Haager Programms weist indes auch Defizite auf.

2009 ist das Jahr eines neuen Europäischen Parlaments, ${ }^{3}$ einer neuen Europäischen Kommission ${ }^{4}$ und möglicherweise sogar einer neuen Vertragsgrundlage der Europäischen Union. Gerade auf dem Feld der Justiz- und Innenpolitik würde der Wegfall der Säulenstruktur durch den Lissabonner Vertrag kompetenziell besondere Auswirkungen haben. Schon einmal, im Jahre 1999, haben Zielvorgaben aus der Kombination einer neuen Vertragsgrundlage, dem in Kraft getretenen Amsterdamer Vertrag, mit dem Mehrjahresprogramm von Tampere eine weitreichende politische Impulswirkung für den Raum der Freiheit, der Sicherheit und des Rechts erzeugt. Die Realitäten einer globalisierten und digitalisierten Welt, des demografischen Wandels und der Überschneidung von innerer und äußerer Sicherheit stellen zudem aktuell besondere Herausforderungen an die Justiz- und Innenpolitik.

Deshalb arbeitet die Europäische Kommission derzeit an einem neuen Programmvorschlag für die europäische Justiz- und Innenpolitik ab 2010, der im Jahr 2009 vom Europäischen Rat verabschiedet werden soll. Worin genau liegt aber der Mehrwert einer solchen Programmplanung? Ist sie ein taugliches Mittel der Politiksteuerung? Und wie muss sie aussehen, um Koordination und Kohärenz von Politik zu gewährleisten?

\section{Sinn und Zweck der Programmplanung}

Die Europäische Union ist ein politisches Mehrebenensystem mit einer Vielzahl von Akteuren, Themen und Aufgaben. Ihre gegenwärtige Drei-Säulenstruktur sieht für einzelne Po-

* Dr. Jörg Semmler, Regierungsdirektor im Bundesministerium des Innern; zurzeit nationaler Sachverständiger bei der Europäischen Kommission, Generaldirektion Justiz, Freiheit und Sicherheit, Referat A1 Strategieplanung, Evaluierung und interinstitutionelle Angelegenheiten, Brüssel.

Dieser Beitrag gibt ausschließlich die persönliche Meinung des Verfassers wieder.

1 Haager Programm zur Stärkung von Freiheit, Sicherheit und Recht in der Europäischen Union, in: Amtsblatt der EU, Nr. C 53 vom 3. März 2005, S. 1-14. Das Programm leitet seinen Namen von der niederländischen Ratspräsidentschaft ab.

2 Rat der Europäischen Union: Schlussfolgerungen des Vorsitzes. Europäischer Rat (Tampere) 15. und 16. Oktober 1999, SN 200/99.

3 Der europaweite Wahltermin liegt in der Zeit vom 4. bis 7. Juni 2009, in Deutschland ist der Wahltag der 7. Juni 2009.

4 Die Amtszeit der derzeitigen Kommission läuft bis zum 31. Oktober 2009. 
litikbereiche überdies unterschiedliche Verfahren der Entscheidungsfindung und Rechtsetzung vor. Um diese Komplexität nicht zuletzt für das bessere Verständnis der Bürger zu reduzieren, bedient sich die Europäische Kommission des Instruments der politischen Planung. Hierunter versteht man die Vorwegnahme politischer Zielvorgaben mit der Absicht, durch eine methodische Verknüpfung von Zwecken, Zielen und Mitteln und eine Systematisierung von Handlungsabfolgen dazu beizutragen, die angestrebten Ziele optimal zu erreichen. Politische Planung steht damit in engem Zusammenhang mit der politischen Steuerung als der zielgerichteten und wirkungsorientierten Gestaltung gesellschaftlicher und wirtschaftlicher (Rahmen-)Bedingungen. Mehrjahresprogramme sind Mittel dieser politischen Planung.

Für den Bereich der europäischen Justiz- und Innenpolitik bedeutet dies, dass der Programmplanung vor allem eine Kohärenzfunktion zukommt. Sie soll also eine ,zusammenhängende' Politik gewährleisten. Und dies in zwei Richtungen: Zum einen stimmt sie die europäische Justiz- und Innenpolitik mit übergeordneten Politikzielen (zum Beispiel der Lissabon-Strategie ${ }^{5}$ ) sowie anderen Politikfeldern (zum Beispiel Außen- und Wirtschaftspolitik) ab. Zum anderen gibt sie sektoralen Politikplanungen (zum Beispiel bezüglich Migration $^{6}$, Asyl ${ }^{7}$, Außengrenzen ${ }^{8}$ ) eine gemeinsame Klammer.

Mehrjahresprogramme erfüllen diese Funktion, indem sie politische Ziele (etwa ein gemeinsamer Raum der Freiheit, der Sicherheit und des Rechts) in strategische (Unter-)Ziele herunterbrechen, Handlungsprioritäten setzen und Einzelmaßnahmen vorschlagen.

\section{Die bisherigen Programme}

Die Betrachtung der bisherigen Mehrjahresplanungen im Bereich Justiz und Inneres soll helfen, sich der Antwort auf die eingangs gestellten Fragen zu nähern. Sie soll zugleich verdeutlichen, wie sich Entstehung, Gestalt und Zweck des Programminstruments verändern. Diese Veränderung trägt zum einen dem wachsenden acquis communautaire und zum anderen dem Spannungsverhältnis zwischen supranationalem und dem noch nicht der Gemeinschaftsmethode unterworfenen ,policy making 'im Bereich Justiz und Inneres Rechnung.

\section{Der Wiener Aktionsplan}

Bereits im Vorfeld des Inkrafttretens des Amsterdamer Vertrags ${ }^{9}$ forderten die Staatsund Regierungschefs auf der Tagung des Europäischen Rates in Cardiff am 15. und 16. Juni

5 Rat der Europäischen Union: Schlussfolgerungen des Vorsitzes. Europäischer Rat (Lissabon) 23. und 24. März 2000, SN 100/00.

6 Europäische Kommission: Mitteilung der Kommission an das Europäische Parlament, den Rat, den Europäischen Wirtschafts- und Sozialausschuss und den Ausschuss der Regionen. Eine gemeinsame Einwanderungspolitik für Europa: Grundsätze, Maßnahmen und Instrumente, KOM (2008) 359.

7 Europäische Kommission: Mitteilung der Kommission an das Europäische Parlament, den Rat, den Europäischen Wirtschafts- und Sozialausschuss und den Ausschuss der Regionen. Künftige Asylstrategie. Ein integriertes Konzept für den EU-weiten Schutz, KOM (2008) 360.

8 Europäische Kommission: Mitteilung der Kommission an das Europäische Parlament, den Rat, den Europäischen Wirtschafts- und Sozialausschuss und den Ausschuss der Regionen. Bericht über die Evaluierung und künftige Entwicklung der Agentur FRONTEX, KOM (2008) 67; Mitteilung der Kommission an das Europäische Parlament, den Rat, den Europäischen Wirtschafts- und Sozialausschuss und den Ausschuss der Regionen. Prüfung der Schaffung eines Europäischen Grenzkontrollsystems (EUROSUR), KOM (2008) 68; Mitteilung der Kommission an das Europäische Parlament, den Rat, den Europäischen Wirtschafts- und Sozialausschuss und den Ausschuss der Regionen. Vorbereitung der nächsten Schritte für die Grenzverwaltung in der Europäischen Union, KOM (2008) 69.

9 Vgl. Amtsblatt der EU, Nr. C 340 vom 10. November 1997. Der Vertrag von Amsterdam wurde von den EUStaats- und Regierungschefs am 16. und 17. Juni 1997 verabschiedet und am 2. Oktober 1997 unterzeichnet. 
$1998^{10}$ Kommission und Rat auf, einen Aktionsplan zur bestmöglichen Umsetzung der justiz- und innenpolitischen Bestimmungen des Vertrages vorzulegen. Die Europäische Kommission leistete dazu im Juli 1998 einen ersten Beitrag mit ihrer Mitteilung „Auf dem Weg zu einem Raum der Freiheit, der Sicherheit und des Rechts“. ${ }^{11}$ Auf dieser Grundlage nahm der Ministerrat am 3. Dezember 1998 einen Aktionsplan ${ }^{12}$ an, der wenige Tage später vom Europäischen Rat von Wien gebilligt wurde ${ }^{13}$ und deshalb als Wiener Aktionsplan bezeichnet wird.

Im Mittelpunkt dieses Aktionsplans stehen die Definitionen der konstitutiven Begriffe des Raumes der Freiheit (freier Personenverkehr), der Sicherheit (Kriminalitätsbekämpfung) und des Rechts (gleicher Zugang zum Recht, Zusammenarbeit der Justizbehörden). Daneben enthält der Aktionsplan Handlungsprioritäten mit vagen Zeitvorgaben. Allerdings betont bereits der Europäische Rat von Wien dessen Übergangscharakter, indem er zur näheren Befassung mit dem Thema Justiz- und Innenpolitik auf den Europäischen Rat von Tampere im Oktober 1999 verweist. ${ }^{14}$ Der Wiener Aktionsplan diente damit lediglich der Vorbereitung des Tampere-Programms.

\section{Das Tampere-Programm}

Der am 1. Mai 1999 in Kraft getretene Vertrag von Amsterdam spricht primärrechtlich zum ersten Mal von der Europäischen Union als einem „Raum der Freiheit, der Sicherheit und des Rechts“, den es schrittweise einzurichten gelte. Dieser neuen Zielvorgabe kommt der Europäische Rat von Tampere am 15. und 16. Oktober 1999 nach, indem er sie an die erste Stelle der politischen Agenda der Union setzt und ein ehrgeiziges Programm aufstellt. In den Schlussfolgerungen der Ratspräsidentschaft ${ }^{15}$ wird das Handlungsfeld der europäischen Justiz- und Innenpolitik - auf der Grundlage des Wiener Aktionsplans - erstmalig für einen Zeitraum von fünf Jahren (1999-2004) anhand von vier ,Meilensteinen“ inhaltlich strukturiert: (a) eine gemeinsame Asyl- und Migrationspolitik der Europäischen Union, (b) ein echter europäischer Rechtsraum, (c) unionsweite Kriminalitätsbekämpfung und (d) stärkeres außenpolitisches Handeln.

Bei diesen ,Meilensteinen“ handelt es sich nur um politische Leitlinien und strategische Unterziele mit teilweise zeitlichen Vorgaben. Spezifische legislative, administrative oder operative Maßnahmen enthält das Programm noch nicht. Auch einen Aktionsplan mit detaillierten Einzelakten, wie er später das Haager Programm begleiten soll, kennt das TampereProgramm noch nicht. So fordert der Europäische Rat in dieser ersten Phase des Aufbaus eines Raumes der Freiheit, der Sicherheit und des Rechts noch grundlegend etwa die Erarbeitung eines umfassenden Migrationskonzeptes, die Errichtung eines gemeinsamen europäischen Asylsystems, die Erstellung von Leitfäden zur justiziellen Zusammenarbeit, die

10 Siehe hierzu Rat der Europäischen Union: Europäischer Rat (Cardiff) 15. und 16. Juni 1998. Schlussfolgerungen des Vorsitzes, SN 150/1/98.

11 Europäische Kommission: Mitteilung der Kommission - Auf dem Weg zu einem Raum der Freiheit, der Sicherheit und des Rechts, KOM (1998) 459.

12 Aktionsplan des Rates und der Kommission zur bestmöglichen Umsetzung der Bestimmungen des Amsterdamer Vertrags über den Aufbau eines Raumes der Freiheit, der Sicherheit und des Rechts, in: Amtsblatt der EU, Nr. C 19 vom 23. Januar 1999, S. 1-15.

13 Rat der Europäischen Union: Europäischer Rat (Wien) 11. und 12. Dezember 1998. Schlussfolgerungen des Vorsitzes, SN 300/98.

14 Ebenda, S. 3.

15 Rat der Europäischen Union: Europäischer Rat, 15. und 16. Oktober 1999. 
Einrichtung einer Task Force der europäischen Polizeichefs und einer europäischen Polizeiakademie sowie die Einrichtung von EUROJUST als Koordinationsstelle nationaler Staatsanwaltschaften bis zum Ende des Jahres 2001.

Darüber hinaus betont das Tampere-Programm den Grundsatz der gegenseitigen Anerkennung von Gerichtsurteilen und anderen Entscheidungen von Justizbehörden als Ausgangspunkt eines gemeinsamen Raumes der Freiheit, der Sicherheit und des Rechts. In dieser ersten Phase der gemeinsamen europäischen Justiz- und Innenpolitik ist dieser Grundsatz der gegenseitigen Anerkennung elementarer Ausdruck gemeinschaftlichen Handelns angesichts einer noch sehr geringen Acquis- beziehungsweise Harmonisierungsdichte ${ }^{16}$ in diesem Bereich. Dies gilt vor allem für die noch nicht vergemeinschaftete, dritte Säule‘. Hieraus erwächst die besondere Bedeutung des Grundsatzes der gegenseitigen Anerkennung als Leitprinzip des ersten Mehrjahresprogramms.

Der Zweck des Tampere-Programms bestand also in erster Linie darin, das politische Ziel der Europäischen Union, einen Raum der Freiheit, der Sicherheit und des Rechts zu schaffen, mit grundsätzlichem Inhalt zu füllen. Das Tampere-Programm hat gegenüber den Mitgliedstaaten einen ersten Impuls für eine gemeinsame europäische Justiz- und Innenpolitik gesetzt und es hat durch die Festlegung von inhaltlichen Prioritäten einen Rahmen für eine kohärente Politik auf diesem Feld geschaffen. ${ }^{17}$

\section{Von Tampere nach Den Haag}

Schon im Halbzeitbericht der belgischen Ratspräsidentschaft zum Programm von Tampere vom 30. Oktober 2001 wurden Umsetzungsdefizite auf dem Weg zu einem gemeinsamen europäischen Justiz- und Innenraum klar identifiziert: zögerliche Rechtsharmonisierung durch die Mitgliedstaaten selbst in Bereichen, die durch den Amsterdamer Vertrag vergemeinschaftet worden waren (Einwanderung, Asyl, Außengrenzen), die Einstimmigkeitsregel als Hindernis im Bereich der ,dritten Säule', Schwierigkeiten der Kompetenzabgrenzung zwischen der Europäischen Union und den Mitgliedstaaten bei der justiziellen Zusammenarbeit in Strafsachen, Verzögerungen bei der Implementierung von Instrumenten der ,dritten Säule' durch die Mitgliedstaaten sowie mangelnder Informationsaustausch zwischen den Mitgliedstaaten und Europol. ${ }^{18}$ Vor diesem Hintergrund unterstrich der Europäische Rat von Laeken am 14. und 15. Dezember 2001 noch einmal die politischen Orientierungen und Ziele, die in Tampere festgelegt worden waren, und forderte zugleich neue Impulse und Leitlinien, um eingetretene Verzögerungen, namentlich bei der Umsetzung europäischer Beschlüsse in innerstaatliches Recht und deren Implementierung, aufzuholen. ${ }^{19}$

Nachdem die Europäische Kommission dem Rat, auf dessen Aufforderung, ${ }^{20}$ bereits zweimal jährlich sogenannte ,Fortschrittsanzeiger' auf dem Weg zu einem Raum der Freiheit, der Sicherheit und des Rechts vorgelegt hatte, nahm sie die letzte dieser halbjährlichen

16 Siehe hierzu Rat der Europäischen Union: Bewertung der Schlussfolgerungen des Europäischen Rates von Tampere, Dok. 13416/01.

17 Siehe hierzu Europäische Kommission: Mitteilung der Kommission an den Rat und das Europäische Parlament. Raum der Freiheit, der Sicherheit und des Rechts: Bilanz des Tampere-Programms und Perspektiven, KOM (2004) 401.

18 Rat der Europäischen Union: Bewertung der Schlussfolgerungen des Europäischen Rates von Tampere, 2001.

19 Rat der Europäischen Union: Schlussfolgerungen des Vorsitzes. Europäischer Rat (Laeken) 14. und 15. Dezember 2001, SN 300/1/01.

20 Rat der Europäischen Union: Europäischer Rat, 15. und 16. Oktober 1999, Einleitung. 
Bestandsaufnahmen im Juni 2004 zum Anlass einer Gesamtbilanz zum Tampere-Programm: ${ }^{21}$

Danach war es zwar grundsätzlich gelungen, die Justiz- und Innenpolitik als für die Bürger vorrangiges Handlungsfeld der Europäischen Union zu etablieren. Gleichwohl waren viele Tampere-Ziele nach Ansicht der Europäischen Kommission noch nicht erreicht worden, etwa in den Bereichen Grundrechtsschutz, EU-Staatsbürgerschaft, integriertes Grenzschutzsystem/Visumpolitik, Steuerung der Migrationsströme, einheitliches Asylverfahren, Aufbau eines europäischen Rechtsraumes, polizeiliche und zollbehördliche Zusammenarbeit sowie Verbrechensprävention und -bekämpfung. Deshalb verband die Kommission ihre Bilanzmitteilung mit spezifischen Überlegungen zu künftigen Prioritäten und Maßnahmen für den Zeitraum bis 2009 und machte ihre Mitteilung zur Grundlage einer öffentlichen Konsultation zum neuen Mehrjahresprogramm. Nach Überzeugung der Kommission sollten die Maßnahmen der Europäischen Union in einem zweiten europäischen Programm für einen Raum der Freiheit, der Sicherheit und des Rechts fortgesetzt und konkretisiert werden, das inhaltliche Prioritäten mit genauen Fristen vorgab. Die Programmplanung von Tampere hatte sich bewährt und sollte grundsätzlich beibehalten werden. Als einen wesentlichen Grund für die Defizite bei der Umsetzung des Tampere-Programms sah die Kommission den institutionellen Rahmen an, der sich mit Inkrafttreten des mittlerweile überholten Verfassungsvertrages und der damit einhergehenden Aufhebung der Säulenarchitektur sowie der Ausweitung der Gemeinschaftsmethode auf den gesamten Justiz- und Innenbereich ändern sollte. 22

\section{Das Haager Programm}

Hatte das Tampere-Programm den Grundstein für einen europäischen Raum der Freiheit, der Sicherheit und des Rechts gelegt, so war das Haager Programm seinem weiteren Ausbau gewidmet.

Vor dem Hintergrund der Evaluierung der Kommission, den Ambitionen des am 29. Oktober 2004 unterzeichneten Verfassungsvertrages sowie der Empfehlung des Europäischen Parlaments zur Anwendung der qualifizierten Mehrheit und des Mitentscheidungsverfahrens $^{23}$ verabschiedete der Europäische Rat am 5. November 2004 das sogenannte Haager Programm, ${ }^{24}$ das zweite Mehrjahresprogramm ,zur Stärkung von Freiheit, Sicherheit und Recht in der Europäischen Union“ (eigene Hervorhebung) für den Zeitraum 2005-2009. In

21 Europäische Kommission: Bilanz des Tampere-Programms und Perspektiven, 2004, Fn. 1. Diese Mitteilung wurde von zwei Arbeitsdokumenten begleitet, einer bereichsspezifischen Bilanzierung sowie einer Übersicht über die wichtigsten im Rahmen des Tampere-Programms angenommenen Rechtsakte. Europäische Kommission: Arbeitsdokument der Kommissionsdienststellen. Anhang zur Mitteilung Der Kommission. Raum der Freiheit, der Sicherheit und des Rechts: Bilanz des Tampere-Programms und Perspektiven, SEK (2004) 693; Commission Staff Working Paper. The Area of Freedom, Security and Justice: assessment of the Tampere programme and future orientations - List of the most important instruments adopted, SEC (2004) 680.

22 Europäische Kommission: Mitteilung der Kommission. Eine Verfassung für die Union. Stellungnahme der Kommission gemäß Artikel 48 des Vertrages über die Europäische Union zum Zusammentritt einer Konferenz von Vertretern der Regierungen der Mitgliedstaaten im Hinblick auf eine Änderung der Verträge, KOM (2003) 548.

23 Europäisches Parlament: Zukunft des Raumes der Freiheit, der Sicherheit und des Rechts. Empfehlung des Europäischen Parlaments an den Rat und den Europäischen Rat zur Zukunft des Raumes der Freiheit, der Sicherheit und des Rechts sowie zu den Bedingungen für die Stärkung seiner Legitimität und Effizienz (2004/ 2175(INI)), P6_TA(2004)0022 vom 14. Oktober 2004; Europäisches Parlament: Bericht des Ausschusses für bürgerliche Freiheiten, Justiz und Inneres, A6-0010/2004 vom 29.09.2004, Berichterstatter Jean-Louis Bourlanges.

24 Rat der Europäischen Union: Tagung des Europäischen Rates (Brüssel), 4. und 5. November 2004. Schlussfolgerungen des Vorsitzes, Dok. 14292/1/04. 
seinen Schlussfolgerungen forderte der Europäische Rat - anders als noch beim Programm von Tampere - die Kommission auf, auf der Grundlage des Haager Programms 2005 einen Aktionsplan mit Vorschlägen für konkrete Maßnahmen und einen Zeitplan für deren Annahme und Umsetzung zu erstellen.

Das Haager Programm unterscheidet sich von seinem Vorläufer in mehrfacher Hinsicht. Schon seine Gliederung bringt zum Ausdruck, dass es nicht mehr darum geht, das Feld der Justiz- und Innenpolitik grundsätzlich zu etablieren und thematisch zu definieren, sondern vielmehr um konkrete weitergehende Maßnahmen zur Erreichung der strategischen Ziele, der Stärkung von (1) Freiheit, (2) Sicherheit und (3) Recht im Rahmen des gemeinsamen europäischen Justiz- und Innenraumes. Das Haager Programm legt als Programm der ,zweiten Generation " einen besonderen Akzent auf die Evaluierung der Umsetzung und Auswirkungen vorangegangener Maßnahmen. Das Instrument der Evaluierung erfährt zudem durch das Haager Programm eine ganz grundsätzliche Aufwertung. Dies erklärt sich unter anderem aus der festen Absicht der Europäischen Union, bei dem europapolitisch noch jungen und zugleich thematisch so vielfältigen und komplexen Politikbereich Justiz und Inneres den Überblick über Handlungsbedarfe zu erhalten und zugleich Verantwortlichkeiten - etwa für Umsetzungsdefizite - klar zuzuweisen. Allerdings sieht das Haager Programm im Gegensatz zum Tampere-Programm nicht mehr halbjährliche, an den Ratspräsidentschaftswechsel gekoppelte, sondern jährliche Fortschrittsanzeiger vor. Die Kommission ist dem Handlungsauftrag des Haager Programms nach umfassender Evaluierung mit einer entsprechenden Mitteilung 25 über einen allgemeinen Evaluierungsmechanismus für den Bereich Freiheit, Sicherheit und Recht im Juni 2006 nachgekommen, die aber vom Ministerrat bisher nicht aufgegriffen worden ist.

Darüber hinaus ist das Haager Programm in vielfacher Hinsicht umfassender, detaillierter und konkreter als sein Vorläufer. Es enthält eine Vielzahl eindeutiger Fristsetzungen und stellt vielfach neben politischen Zielen und Forderungen auch klare Kriterien und Voraussetzungen für deren Umsetzung auf, etwa im Bereich der Visumpolitik, des polizeilichen Informationsaustauschs und der Terrorismusbekämpfung. Ein weiterer Aspekt belegt den Charakter des Haager Programms als den eines Programms der ,zweiten Generation“: Es handelt sich um den Grundsatz der Verfügbarkeit, jenes innovative Konzept für den grenzüberschreitenden Austausch von strafverfolgungsrelevanten Informationen. Auf der Grundlage eines mittlerweile existierenden rechtlichen, technischen und operativen Integrationsbestands im Bereich Justiz und Inneres ist der Grundsatz der Verfügbarkeit zum Leitprinzip des Haager Programms geworden.

Noch in einem anderen Punkt unterscheiden sich beide Programme substanziell: dem Bereich der externen Dimension des Raumes der Freiheit, der Sicherheit und des Rechts. Hatte noch das Tampere-Programm lediglich davon gesprochen, dass „die Anliegen im Bereich Justiz und Inneres [...] bei der Festlegung und Durchführung anderer Politiken und Maßnahmen der Union einbezogen werden müssen“, ${ }^{26}$ so sieht das Haager Programm das „Bestehen einer internen Politik als wichtigsten Parameter für die Begründung einer externen Maßnahme“. ${ }^{27}$ Darüber hinaus erhielt die Kommission im Haager Programm gemeinsam mit dem Generalsekretär des Rates und Hohen Vertreter für die Gemeinsame Außen- und Sicherheitspolitik den Auftrag zur Erstellung einer umfassenden Strategie zu allen externen

25 Europäische Kommission: Mitteilung der Kommission an den Rat und das Europäische Parlament. Evaluierung der EU-Maßnahmen im Bereich Freiheit, Sicherheit und Recht, KOM (2006) 332.

26 Rat der Europäischen Union: Europäischer Rat, 15. und 16. Oktober 1999, S. 59.

27 Haager Programm, 2005, S. 14. 
Aspekten der Unionspolitik für Freiheit, Sicherheit und Recht bis Ende des Jahres 2005. ${ }^{28}$ Dieser Bedeutungszuwachs der außenpolitischen Dimension der europäischen Justiz- und Innenpolitik war nicht zuletzt eine Folge der weltweiten Sicherheitslage nach den Terroranschlägen vom 11. September 2001 (New York), 11. März 2004 (Madrid) und 7. Juli 2005 (London).

Der Aktionsplan zum Haager Programm: Ausdruck des größten qualitativen Unterschieds beider Mehrjahresprogramme im Hinblick auf die konkrete Politiksteuerung ist der sogenannte Aktionsplan für die Jahre 2005-2009, zu dessen Aufstellung die Kommission im Haager Programm aufgefordert worden war. Diesem Auftrag kam die Kommission im Mai 2005 mit ihrer Mitteilung „Das Haager Programm: Zehn Prioritäten für die nächsten fünf Jahre" ${ }^{29}$ nach. Der Europäische Rat bekräftigte dann am 16./17. Juni 2005 den aus dieser Mitteilung hervorgegangenen Aktionsplan. ${ }^{30}$

Dieser besteht aus zwei Teilen. In einem ersten Schritt leitet er - neben einem Überblick über die Problemstellungen und wichtigsten Aspekte des Haager Programms - zehn prioritäre Handlungsfelder aus den drei strategischen Programmzielen Stärkung der Freiheit, Stärkung der Sicherheit und Stärkung des Rechts ab. In einem zweiten Schritt bricht er diese prioritären Handlungsfelder in einen Katalog von insgesamt über 260 konkreten Einzelvorhaben, Initiativen und Aktionen herunter. Diese Maßnahmen reichen von Rechtsetzungsvorschlägen und Grünbüchern, über Fortschrittsberichte, Studien und Best-Practice-Leitfäden bis hin zu Einzelprogrammen und Finanzmaßnahmen. Dieser Aufgabenkatalog zeichnet sich nicht nur thematisch, sondern auch methodisch durch eine enorme Konkretisierung und Detailliertheit aus; hinzukommt, dass für jedes einzelne Listenvorhaben ein klares Zeitziel für dessen Annahme und Umsetzung vorgegeben ist. Damit besteht zum ersten Mal ein umfassendes, konkretes Arbeitsprogramm für die europäische Justiz- und Innenpolitik, das Aufgaben sowohl auf EU- als auch auf nationaler Ebene vorsieht. Dieses Arbeitsprogramm ist zugleich Ausdruck des in gut fünf Jahren seit seiner Initiierung erheblich angewachsenen acquis communautaire im Raum der Freiheit, der Sicherheit und des Rechts, der mittlerweile 512 Rechtsakte umfasst. ${ }^{31}$

Die Bewertung der Umsetzung des Haager Programms und seines Aktionsplans: Vor dem Hintergrund des ursprünglich für den 1. November 2006 vorgesehenen Inkrafttretens des Verfassungsvertrages hatte der Europäische Rat im November 2004 sich selbst für die zweite Hälfte des Jahres 2006 zu einer Prüfung der Fortschritte beim Haager Programm verpflichtet sowie der Kommission den Auftrag zu jährlichen Berichten über den Stand der Umsetzung der Maßnahmen, sogenannte ,Fortschrittsanzeiger" (, scorebords"), erteilt. Darüber hinaus hatte der Rat die Kommission im Haager Programm aufgefordert, ihm bis zum vorgesehenen Inkrafttreten des Verfassungsvertrages über die erzielten Fortschritte gesondert Bericht zu erstatten und Vorschläge für die erforderliche Ergänzung des Programms zu unterbreiten. ${ }^{32}$

28 Europäische Kommission: Mitteilung der Kommission. Eine Strategie für die Außendimension des Raumes der Freiheit, der Sicherheit und des Rechts, KOM (2005) 491.

29 Europäische Kommission: Mitteilung der Kommission an den Rat und das Europäische Parlament. Das Haager Programm: Zehn Prioritäten für die nächsten fünf Jahre. Die Partnerschaft zur Erneuerung Europas im Bereich der Freiheit, der Sicherheit und des Rechts, KOM (2005) 184.

30 Rat der Europäischen Union: Europäischer Rat (Brüssel) 16. und 17. Juni 2005. Schlussfolgerungen des Vorsitzes, Dok. 10255/1/05. Begleitet wird dieser Aktionsplan von der im Dezember 2004 gesondert verabschiedeten Europäischen Drogenstrategie für den Zeitraum 2005-2012 sowie zwei dazugehörigen EUDrogenaktionsplänen; Rat der Europäischen Union: EU-Drogenstrategie (2005-2012), Dok. 15074/04; EUDrogenaktionsplan (2005-2008), in: Amtsblatt der EU, Nr. C 168 vom 8. Juli 2005, S. 1-18; EU-Drogenaktionsplan (2009-2012), in: Amtsblatt der EU, Nr. C 326 vom 20. Dezember 2008, S. 7-25.

31 Siehe http://eur-lex.europa.eu/de/legis/20081001/chap19.htm (letzter Zugriff: 15.12.2008).

32 Siehe insgesamt Rat der Europäischen Union: Europäischer Rat, 4. und 5. November 2004. 
Die Kommission kam ihrem Auftrag im Juni 2006 mit einem Paket von Bestandsaufnahme-Mitteilungen nach: Im Mittelpunkt stand die Mitteilung „Umsetzung des Haager Programms: Weitere Schritte" 33 mit einer ersten umfassenden politischen Bewertung der bei der Umsetzung des Haager Programms erzielten Fortschritte und Vorschlägen für notwendige Anpassungen. Begleitet wurde diese Mitteilung vom ersten Fortschrittsanzeiger „Bericht über die Umsetzung des Haager Programms im Jahr 2005“, der eine Messung des Grades der Umsetzung der im Aktionsplan vorgesehenen Maßnahmen auf EU- und nationaler Ebene vorsah. Des Weiteren stellte die Kommission in diesem Kontext auch die bereits erwähnte Mitteilung zu einem generellen Evaluierungsmechanismus justiz- und innenpolitischer Maßnahmen vor. ${ }^{34}$ Auch die finnische Präsidentschaft hatte im November 2006 in einem Halbzeitbericht eine Bewertung des bisherigen Umsetzungsstandes des Haager Programms vorgenommen und darausfolgende Handlungsprioritäten für die zweite Programmhälfte identifiziert. ${ }^{35}$ Die daraufhin im Dezember 2006 auf der Grundlage der Bewertungen der Kommission und der Ratspräsidentschaft verabschiedeten Schlussfolgerungen des Ministerrats hoben vor allem die Defizite in der justiziellen Zusammenarbeit in Strafsachen und der polizeilichen Zusammenarbeit sowie hinsichtlich der Effizienz der Beschlussfassung im Ministerrat hervor. ${ }^{36}$ Der Europäische Rat bekräftigte schließlich auf seiner Dezembertagung 2006 die Schlussfolgerungen des Ministerrates und unterstrich zuvörderst die Bedeutung einer umfassenden europäischen Migrationspolitik als eine der Hauptprioritäten für die Europäische Union am Beginn des 21. Jahrhunderts. ${ }^{37}$

Alle diese Bewertungen kommen zu dem Ergebnis, dass der weitere Ausbau des Raumes der Freiheit, der Sicherheit und des Rechts eines neuen Impulses bedarf. Die jährlichen Fortschrittsanzeiger belegen, dass die Umsetzungsrate der Maßnahmen weit hinter den Vorgaben des Aktionsplans zurückbleibt. Sind 2005 noch 65 Prozent der Maßnahmen des Aktionsplans im vorgesehenen Zeitplan umgesetzt worden, so waren es 2006 noch 53 Prozent und 2007 nur noch 38 Prozent. ${ }^{38}$ Dabei ist die Umsetzungsrate in der noch nicht vergemeinschafteten ,dritten Säule' im Verhältnis geringer als in den vergemeinschafteten Bereichen der ,ersten Säule‘. Zwar sieht der Aktionsplan die überwiegende Zahl der Maßnahmen für die Jahre 2005-2007 vor, doch ist die Diskrepanz zwischen Vorgabe und Programmerfüllung so beträchtlich, dass auch bis 2009 nicht alle Zielvorgaben erreicht werden können. Dies ist nicht zuletzt den nach wie vor schwerfälligen Entscheidungsstrukturen im Minister-

33 Europäische Kommission: Mitteilung der Kommission an den Rat und das Europäische Parlament. Umsetzung des Haager Programms: Weitere Schritte, KOM (2006) 331.

34 Europäische Kommission: Evaluierung der EU-Maßnahmen im Bereich Freiheit, Sicherheit und Recht, 2006. Auch die Mitteilung die Gewährleistung eines effektiveren gerichtlichen Rechtsschutzes betreffend gehört zu diesem Paket. Europäische Kommission: Mitteilung der Kommission an das Europäische Parlament, den Rat, den Europäischen Wirtschafts- und Sozialausschuss, den Ausschuss der Regionen und den Gerichtshof der Europäischen Gemeinschaften über die Anpassung der die Zuständigkeit des Gerichtshofs betreffenden Bestimmungen des Titels IV des Vertrags zur Gründung der Europäischen Gemeinschaft im Hinblick auf die Gewährleistung eines effektiveren gerichtlichen Rechtsschutzes, KOM (2006) 346.

35 Rat der Europäischen Union: Reviewing The Hague Programme: a new impetus to strengthen the Area of Freedom, Security and Justice, Dok. 15844/06.

36 Rat der Europäischen Union (Justiz und Inneres): Überprüfung des Haager Programms - Schlussfolgerungen des Rates, 4./5. Dezember 2006, Dok. 16340/06.

37 Rat der Europäischen Union: Europäischer Rat (Brüssel) 14. und 15. Dezember 2006. Schlussfolgerungen des Vorsitzes, Dok. 16879/1/06.

38 Siehe hierzu die Berichte über die Umsetzung des Haager Programms der Jahre 2005, 2006 und 2007. Europäische Kommission: Mitteilung der Kommission an den Rat und das Europäische Parlament. Bericht über die Umsetzung des Haager Programms im Jahr 2005, KOM (2006) 333; Mitteilung der Kommission an den Rat und das Europäische Parlament. Bericht über die Umsetzung des Haager Programms im Jahr 2006, KOM (2007) 373; Mitteilung der Kommission an den Rat und das Europäische Parlament. Bericht über die Umsetzung des Haager Programms im Jahr 2007, KOM (2008) 373. 
rat geschuldet, deren Vereinfachung maßgeblich vom Inkrafttreten des Lissabonner Reformvertrages abhängt. Auch nach 2009 wird die Europäische Union also Initiativen und Maßnahmen des Haager Programms fortführen müssen, will sie die Einhaltung der festgelegten strategischen Prioritäten für den Ausbau des Raumes der Freiheit, der Sicherheit und des Rechts nicht gefährden.

In jedem Fall aber hat das Haager Programm einen wesentlichen Beitrag dazu geleistet, dass dieser Ausbau arbeitsteilig, strukturiert, nachhaltig, evaluierbar und vor allem kohärent erfolgen kann.

\section{Das Instrument der Mehrjahresprogramme}

Wie kaum ein anderer Politikbereich betrifft der gemeinsame Raum der Freiheit, der Sicherheit und des Rechts unmittelbar das Alltagsleben der Menschen in der Europäischen Union. Es geht um nicht weniger als die Wahrung der Grundrechte, um Freizügigkeit und Datenschutz, um die Unionsbürgerschaft einschließlich der Teilnahme an Kommunal- und Europawahlen. Es geht um die Bekämpfung grenzüberschreitender, organisierter Kriminalität durch polizeiliche und justizielle Zusammenarbeit ebenso wie um den Kampf gegen Terrorismus und um Drogenbekämpfung. Eine große Herausforderung ist die Steuerung der legalen und die Verhinderung der illegalen Einwanderung. Dasselbe gilt für die Integration von Zuwanderern, die Asyl- und Visumpolitik sowie die Sicherheit der Außengrenzen. Nicht zuletzt geht es auch um die Gewährleistung effektiven Rechtsschutzes im Zivilrecht. Ein gemeinsamer Raum der Freiheit, der Sicherheit und des Rechts muss die Voraussetzungen für offene Gesellschaften in einer globalisierten und in immer stärkerem Maße technisierten Welt schaffen. Daher sieht sich die Europäische Union gerade in diesem Bereich ganz konkreten und wachsenden Erwartungen der Bürgerinnen und Bürger gegenüber.

Die Vertragsgrundlagen der Europäischen Union tragen der besonderen Bedeutung der drei öffentlichen Güter Freiheit, Sicherheit und Recht Rechnung, indem sie sie als strategische Prioritäten in ihre grundlegenden Bestimmungen aufnehmen (Art. 2 EUV). ${ }^{39}$ Eines der wichtigsten Ziele der EU-Politik muss es also sein, die gemeinsame Leistungsfähigkeit der Union und der Mitgliedstaaten zur Gewährleistung dieser drei öffentlichen Güter zu verbessern. Eine mehrjährige strategische Vorausplanung verbunden mit einem konkreten Arbeitsprogramm ist dafür unverzichtbar:

Ein solches Mehrjahresprogramm sichert die Kohärenz der Fülle von Einzelmaßnahmen in einem thematisch so vielfältigen Politikfeld wie der Justiz- und Innenpolitik. Das ist umso wichtiger, als sich viele der Einzelpolitiken dieses Bereichs überschneiden und teilweise auch konfligieren, so etwa Terrorismus- und Kriminalitätsbekämpfung mittels moderner Technologien auf der einen und Datenschutz auf der anderen Seite. Mehrjahresprogramme ermöglichen es, den sektoralen Ansatz von Politikplanung, zum Beispiel im Migrationsoder Terrorismusbereich, mit übergeordneten politischen Zielsetzungen - etwa für die Außendimension der Justiz- und Innenpolitik - in Einklang zu bringen.

Dies gilt auch im Hinblick auf die Vielzahl politischer Akteure, insbesondere im Bereich der , dritten Säule‘. Der kooperative Ansatz in der polizeilichen und justiziellen Zusammenarbeit in Strafsachen zieht einen erhöhten Koordinationsbedarf nach sich, der durch die Programmplanung gewährleistet werden kann. Mehrjahresprogramme bieten die Chance für die Europäische Kommission zusammen mit dem Rat, das gemeinsame europäische Interesse

39 Art. 2 des Vertrags über die Europäische Union (EUV) in der konsolidierten Fassung des Vertrags von Nizza. (Amtsblatt der EU, Nr. C 325 vom 24. Dezember 2002, S. 5-32). Siehe auch Art. 3 Abs. 2 EUV in der konsolidierten Fassung des Vertrags von Lissabon (Amtsblatt der EU, Nr. C. 115 vom 9. Mai 2008). 
im Raum der Freiheit, der Sicherheit und des Rechts zu destillieren und zu definieren. Dieses Ergebnis stellt zudem eine gute Grundlage dar für eine strukturierte Debatte von Kommission und Rat mit dem Europäischen Parlament über die künftige Justiz- und Innenpolitik und ihre Auswirkungen auf Bürger und Mitgliedstaaten mit dem Ziel eines inter-institutionellen Einvernehmens über die grundlegenden zukünftigen Weichenstellungen.

Das Programm von Tampere hat bewiesen, dass das Instrument der Rahmenprogramme am besten geeignet ist, politische Impulse zu setzen und eine allgemeine Vorstellung von künftigen Prioritäten zu entwickeln. Die Wirkung der letztendlichen Prioritätensetzung durch den Europäischen Rat der Staats- und Regierungschefs, der die Mehrjahresprogramme im Bereich Justiz und Inneres wegen ihrer grundsätzlichen politischen Bedeutung bisher verabschiedet hat, ist dabei nicht zu vernachlässigen. Die oben erwähnten Bestandsaufnahmen der Europäischen Kommission sowie der finnischen Ratspräsidentschaft zum Haager Programm unterstreichen nachdrücklich, dass es einer solchen neuen Impulssetzung in der europäischen Justiz- und Innenpolitik dringend bedarf. Das Jahr 2009 scheint als Zeitpunkt für einen solchen Impuls günstig. Sowohl im Jahre 1999 als auch im Jahre 2004 standen die Impulssetzungen des Tampere- und des Haager Programms für den gemeinsamen Raum der Freiheit, der Sicherheit und des Rechts im Zusammenhang mit grundlegenden Vertragsänderungen. Im ersten Fall mit dem im Mai 1999 in Kraft getretenen Amsterdamer Vertrag, im zweiten mit dem im Oktober 2004 unterzeichneten Verfassungsvertrag. Möglicherweise bietet das Jahr 2009 mit dem Inkrafttreten des Lissabonner Reformvertrags einen ähnlichen Resonanzboden für ein neues Mehrjahresprogramm im Bereich Justiz und Inneres.

Das Haager Programm hat gezeigt, dass inhaltliche Konsistenz und Nachhaltigkeit europäischer Justiz- und Innenpolitik nur auf der Grundlage einer umfassenden und konkreten Programmplanung möglich ist. Nachhaltige Politik basiert auf der verlässlichen Evaluierung vorangegangener und begonnener Maßnahmen. Hierfür ist ein detaillierter Arbeitsplan mit Zeitzielen unerlässlich, dessen Einhaltung regelmäßig überprüft werden muss. Dies sichert den Überblick über Handlungsbedarfe, ermöglicht nachträgliche Justierungen und ist damit letztlich unentbehrliches Instrument einer effizienten Koordination. Die sogenannten ,Fortschrittsanzeiger" der Europäischen Kommission haben sich in dieser Hinsicht sehr bewährt. Allerdings darf ein solcher Arbeitsplan kein Hindernis für eine flexible Reaktion auf unvorhergesehene Ereignisse - wie etwa terroristische Anschläge oder Flüchtlingsströme - darstellen. Darüber hinaus strukturiert Programmplanung die Politik, indem sie zwischen strategischen Zielen, Handlungsprioritäten und Einzelmaßnahmen unterscheidet. Sie ist also der Versuch, den komplexen politischen Prozess in der Europäischen Union zumindest ein Stück weit zu rationalisieren.

Das Steuerungsinstrument der Programmplanung ist auch in anderen Politikbereichen der Europäischen Union durchaus üblich. Als derzeit ${ }^{40}$ wohl prominentestes Beispiel sei hier die Lissabon-Strategie ${ }^{41}$ angeführt, mit der sich die Europäische Union das ehrgeizige Ziel gesetzt hat, die Union bis 2010 zum wettbewerbsfähigsten und dynamischsten wissensbasierten Wirtschaftsraum in der Welt zu machen. Es handelt sich hierbei um eine Meta-Rahmenplanung, die alle Politiken der Europäischen Union umfasst. Eines ihrer Unterziele ist es, die europäische Rechtsetzung insgesamt transparenter, einfacher und schlicht qualitativ

40 Historisch von besonderer Bedeutung ist in dieser Hinsicht das Weißbuch der Delors-Kommission zur Verwirklichung des Binnenmarktes vom 14. Juni 1985. Europäische Kommission: Vollendung des Binnenmarktes: Weißbuch der Kommission an den Europäischen Rat, KOM (85) 310.

41 Rat der Europäischen Union: Europäischer Rat, 23. und 24. März 2000. 
besser zu machen (sogenannte , better regulation'). Auch diesem Ansatz dient ein Mehrjahresprogramm im Justiz- und Innenbereich, da es durch seine Kohärenz-, Konsistenz- und Koordinationsanforderungen grundsätzlich auf verhältnismäßige Rechtsetzung zielt.

Vor diesem Hintergrund hat schließlich auch der Lissabonner Reformvertrag den guten Erfahrungen mit den beiden bisherigen Mehrjahresprogrammen sowie der Bedeutung der Programmplanung für die Steuerung der europäischen Justiz- und Innenpolitik dadurch Rechnung getragen, dass er sie ausdrücklich in Art. 68 des Vertrages über die Arbeitsweise der Europäischen Union erwähnt. Dort heißt es: „Der Europäische Rat legt die strategischen Leitlinien für die gesetzgeberische und operative Programmplanung im Raum der Freiheit, der Sicherheit und des Rechts fest.“42

Dieses planerische Politikverständnis der Europäischen Union folgt einem wissenschaftlichen Politikansatz, der etwa in den Instrumenten der für die Europäische Kommission typischen Grün- und Weißbücher seinen Ausdruck findet. Im Kern geht es darum, zuerst eine politische Idee zu entwickeln, um dann darauf gegründete konkrete Rechtsetzungsvorhaben zu planen. Diese Art der politischen ,Vorausplanung' kontrastiert mit der politischen Arbeitsweise in den Mitgliedstaaten. In Deutschland zum Beispiel sind Ansätze solcher politischen Planung lediglich in dem Instrument der mittelfristigen Finanzplanung nach Art. 109 Abs. 3 Grundgesetz oder etwa in Koalitionsverträgen zu erkennen. Überdies widerlegen die Programmplanungen der Europäischen Union die ihr gegenüber häufig geäußerte Kritik der Intransparenz politischer Entscheidungsfindung, indem sie frühzeitig Chancen für Diskussion und Kritik eröffnen.

\section{Ausblick}

Die genannten Argumente belegen die Sinnhaftigkeit und Notwendigkeit eines neuen Mehrjahresprogramms als Steuerungsinstrument für eine gemeinsame europäische Justizund Innenpolitik der Jahre 2010-2014. Es kommt hinzu, dass bereits in vielfältiger Weise Überlegungen zu einer neuen strategischen Vision für diesen Bereich stattfinden. Ein wesentlicher Beitrag sind in diesem Zusammenhang die Empfehlungen der beiden „Hochrangigen beratenden Gruppen zur Zukunft der europäischen Justiz- und Innenpolitik“, die sogenannten „Zukunftsgruppen“, die der Kommission vom Ministerrat im Juli 2008 als Reflexionsgrundlage übermittelt worden sind. ${ }^{43}$ Die Europäische Kommission hat von September bis Dezember 2008 eine umfassende öffentliche Internetkonsultation zur Zukunft der europäischen Justiz- und Innenpolitik durchgeführt, deren Ergebnisse ebenfalls in ihre Zukunftsüberlegungen einfließen werden. ${ }^{44}$ Die Europäische Kommission hat auch bereits die nationalen Parlamente in die Reflexionen über die künftigen Prioritäten in diesem Bereich einbezogen. Das Europäische Parlament ist - nicht zuletzt wegen der Europawahl im Juni 2009 - sehr daran interessiert, bereits in der Vorbereitungsphase des neuen Programms in einen entsprechenden politischen Dialog mit Kommission und Rat zu treten. Dasselbe gilt für die tschechische und die schwedische Ratspräsidentschaft im Jahre 2009.

42 Art. 68 des Vertrags über die Arbeitsweise der Europäischen Union in der konsolidierten Fassung des Vertrags von Lissabon (Amtsblatt der EU, Nr. C 115 vom 9. Mai 2008, S. 47-199).

43 Rat der Europäischen Union: Freiheit, Sicherheit, Schutz der Privatsphäre - Europäische Innenpolitik in einer offenen Welt - Bericht der Informellen Hochrangigen Beratenden Gruppe zur Zukunft der europäischen Innenpolitik (,Zukunftsgruppe“), Dok. 11657/08. Der Abschlußbericht der Zukunftsgruppe Justiz ist nicht veröffentlicht worden.

44 Siehe http://ec.europa.eu/justice_home/news/consulting_public/news_consulting_0001_en.htm (letzter Zugriff: 15.12 .2008$)$. 
Die Europäische Kommission erarbeitet gegenwärtig einen Entwurf für ein neues Mehrjahresprogramm für den Zeitraum 2010-2014, den sie zusammen mit einer umfassenden Evaluierung des Haager Programms im Mai 2009 dem Rat der Justiz- und Innenminister sowie der Öffentlichkeit vorstellen wird. Im Anschluss daran wird es auf dieser Grundlage voraussichtlich einen Gedankenaustausch mit dem Europäischen Parlament geben und der Kommissionsentwurf wird in den unterschiedlichen Ratsgremien verhandelt. Es ist sehr wahrscheinlich, dass die schwedische Präsidentschaft auf eine Verabschiedung des ,PostHaager-Programms ‘ bis zum Ende des Jahres 2009 hinarbeiten wird.

Dieses neue Mehrjahresprogramm wird vor allem Antworten finden müssen auf die immer stärkere Überschneidung von europäischer Innen- und Außenpolitik. Dies gilt umso mehr, als die bereits erwähnte Strategie für die Außendimension des Raumes der Freiheit, der Sicherheit und des Rechts ebenfalls im Jahr 2009 ausläuft. Es sollte zudem einen sensiblen Ausgleich bieten zwischen dem Einsatz neuer Technologien zu Sicherheitszwecken und den bürgerrechtlichen Anforderungen an Datenschutz und Privatsphäre. Nicht zuletzt wird es die Vorgaben des Migrations- und Asylpakts in konkrete Handlungsanforderungen für die kommenden Jahre herunterbrechen müssen.

Als Mehrjahresprogramm der ,dritten Generation “ wird es auf die Vorläuferprogramme aufbauen. Möglicherweise wird sein Schwerpunkt weniger auf legislativen Vorgaben liegen und vielmehr Implementierungsmaßgaben enthalten. Bessere Zusammenarbeit, Koordination, Netzwerke, Plattformen und Interoperabilität werden voraussichtlich zu den neuen Schlagworten zählen. Das grundlegende Prinzip dieses neuen - bisweilen bereits als ,Stockholmer" bezeichneten - Programms wäre dann das der „Konvergenz“. 45

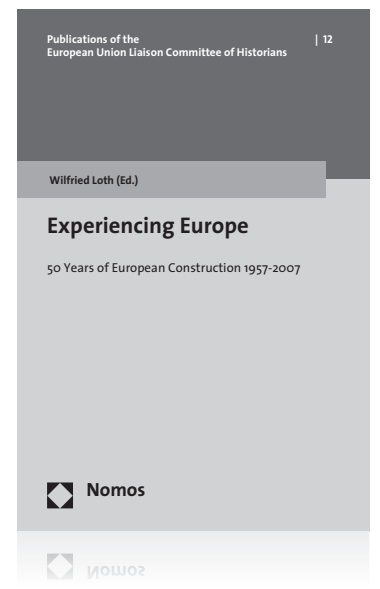

Bitte bestellen Sie im Buchhandel oder versandkostenfrei unter $>$ www.nomos-shop.de

\section{Experiencing Europe} 50 Years of European Construction 1957-2007 Herausgegeben von Prof. Dr. Wilfried Loth 2009, 362 S., brosch., 59,- €, ISBN 978-3-8329-4124-6 (Veröffentlichungen der Historiker-Verbindungsgruppe bei der Kommission der EG, Bd. 12)

19 bekannte EU-Historiker aus sieben Ländern bilanzieren die verschiedenen Aspekte der europäischen Integration. Auf breite Quellenstudien gestützt bietet der Band zum ersten Mal eine kohärente europäische Perspektive auf die Integrationsgeschichte - darüber hinaus diskutieren die Beiträge die gegenwärtige Situation und mögliche Entwicklungen im Licht dieser Bilanz.

45 Rat der Europäischen Union (Justiz und Inneres): Entwurf von Schlussfolgerungen des Rates zum Konvergenzprinzip und zur Struktur der inneren Sicherheit, Dok. 14069/08. 